\title{
Orion Cislunar Guidance and Navigation
}

\author{
Christopher D'Souza ${ }^{*}$, Timothy Crain. ${ }^{\dagger}$ \\ NASA Johnson Space Center, Houston, TX 77058, USA \\ and \\ Fred C. Clark \\ Charles Stark Draper Laboratory, Houston, TX 77058, USA
}

\begin{abstract}
The Orion vehicle is being designed to provide nominal crew transport to the lunar transportation stack in low Earth orbit, crew abort prior during transit to the moon, and crew return to Earth once lunar orbit is achieved. Design of guidance and navigation algorithms to perform maneuvers in support of these functions is dependent on the support provided by navigation infrastructure, the performance of the onboard GN\&C system, and the choice of trajectory maneuver methodology for outbound and return mission phases. This paper documents the preliminary integrated analyses performed by members of the Orion Orbit GN\&C System team investigating the navigation update accuracy of a modern equivalent to the Apollo era ground tracking network and the expected onboard dispersion and navigation errors during a lunar mission using a linear covariance error analysis technique.
\end{abstract}

\section{Introduction}

$\mathrm{T}$ he Orion vehicle is being designed to provide nominal crew transport to the lunar transportation stack in low Earth orbit, crew abort prior during transit to the moon, and crew return to Earth once lunar orbit is achieved. Design of guidance and navigation algorithms to perform maneuvers in support of these functions is dependent on the support provided by navigation infrastructure, the performance of the onboard GN\&C system, and the choice of trajectory maneuver methodology for outbound and return mission phases. This paper documents the preliminary integrated analyses performed by members of the Orion Orbit GN\&C System team investigating the navigation update accuracy of a modern equivalent to the Apollo era ground tracking network and the expected onboard dispersion and navigation errors during a lunar mission using a linear covariance error analysis technique. The STDN navigation analysis is performed only on the transEarth trajectory to establish that a working simulation for Orion analysis can reproduced Apollo equivalent accuracies. This tool will then be used in support of trades for sizing the infrastructure needed to support the upcoming lunar exploration missions. In parallel with this navigation analysis, a the linear covariance study assumes Apollo ground navigation accuracies to analyze both outbound translunar and tranEarth return trajectories. The translunar trajectories are nominally the responsibility of Constellation elements other than Orion, but the dispersions from this mission phase is needed to initialize abort scenario development for the Orion vehicle.

The CEV/LLV stack will be inserted into a translunar orbit by the Earth Departure Stage. Soon after translunar injection (TLI) maneuver the EDS will separate from the CEV/LLV stack. It will perform 4 TCMs during the 3-day coast. Depending on the desired lunar orbit, a sequence of (up to three) maneuvers will be performed to insert the $\mathrm{CEV} / \mathrm{LLV}$ stack into a low lunar orbit, that passes over the landing site. This insertion maneuver sequence could take up to 24 hours. After insertion, the LLV will separate from the now un-crewed CEV, which will continue unmanned operations until the LLV ascent stage returns to dock with the CEV. After the crew and cargo is transferred to the CEV, the LLV will separate from the CEV and the CEV will prepare for the Trans-Earth Injection (TEI) sequence. This could be a three-burn sequence with the transfer orbit between the first and the last maneuver

\footnotetext{
* Aerospace Engineer, EG6, Aeroscience and Flight Mechanics Division, Member AIAA

${ }^{\dagger}$ Orion Orbit GNC Manager, EG6, Aeroscience and Flight Mechanics Division, Member AIAA

* Principal Member of the Technical Staff, Guidance, Navigation and Control Division
} 
allowing for a period of upto 48 hours. After the TEI sequence, the remaining transfer time would be 72 hours to reach the Entry Interface point.

The paper is organized as follows. Section 2 contains a description of the tools used in the design and analysis as well as the assumptions made. Section 3 contains the translunar design and analysis, including the TLI and lunar orbit insertion phase. Section 4 contains the design and analysis of the transEarth phase, including the TEI maneuvers. Finally, Section 5 contains some concluding comments.

\section{Tools and Assumptions}

\section{A. Linear Covariance Analysis and Tools}

Linear covariance analysis is a methodology to obtain the navigation performance (statistics) of a system in a single run! It is a complement to Monte Carlo analysis in that it provides the statistical behavior of a GNC system without the thousands of Monte Carlo runs needed in order to truly represent the statistics of a complex dynamical system. During the early phase of a system design it is invaluable in being able to provide the engineer with the system performance, especially as it relates to navigation and guidance issues. It is used to quickly (and accurately) understand how various navigation sensors affect the navigation accuracy of a particular system design under consideration. Additionally, it also provides trajectory dispersion information (the difference between the actual trajectory and the nominal trajectory) along with variation in fuel (or $\Delta V$ ) usage. Finally, it is the perfect tool in order to trade the performance of different sensors or to determine the contribution of a particular sensor (error) parameter.

However, it is necessary to keep in mind the limitations of linear covariance analysis, particularly as it relates to rendezvous analysis. As the name suggests, it is linear covariance analysis. It relies on the methodology of Kalman filtering (as was originally obtained by Kalman in 1960); it operates on perturbation states, which are assumed to be linear. The nominal dynamics are propagated using the nonlinear dynamics. The measurement sensitivities are the partial derivatives of the nonlinear measurements with respect to the states of interest (as in a Kalman filter). As the second part of the name denotes, this analysis is based upon the covariance matrices associated with the Kalman filter. Recall that within virtually all variants of Kalman filters, the covariance matrices are propagated in a linear fashion (either by means of a state transition matrix or by integrating the Ricatti differential equation). We choose the latter formulation for propagating the covariance matrix. The nominal states are propagated using the full nonlinear equations.

In the cislunar linear covariance analysis we deal with the trajectory dispersion covariance (the covariance of the difference between the actual states and the nominal states), the navigation dispersion covariance (covariance of the difference between the navigated states and the nominal states) and the on-board navigation errors (covariance of the difference between the navigation states and actual states). We carry along and operate on all these three covariance matrices.

\section{Dynamics Models}

For this CEV cislunar analsysis, the gravity fields of the bodies (Sun, Earth and Moon) were assumed to be spherical. However, all three bodies were assumed to be active during the entire trajectory. The integrator used for the propagation of the covariance dynamics was a fourth-order Runge-Kutta method. The trajectory of the CEV was propagated using a (fourth-order) Encke-Nyström integrator. In order to retain precision in the covariance propagation, a 10 second time step was used.

The attitude was modeled as a right-handed quaternion (with scalar first). For the covariance associated with the attitude dynamics, the Bortz formulation was used. Unlike the quaternion representation in a Kalman filter, it provides for an attitude covariance matrix of full rank without any approximations.

For this early analysis, the attitude control system was not modeled. Once again, this can be incorporated as the system matures. Particularly for this phase of the mission, the attitude control was not considered to be particularly important when PTC (Passive Thermal Control, a.k.a. the barbeque roll) will, most likely, be used.

One of the significant factors in the translunar trajectory is the translation noise. This manifests itself in imperfectly coupled rotational (attitude) maneuvers, venting, etc. In the filter this is modeled as process noise and is one of the parameters that is varied. There are two types of periods modeled: active and quiescence. For the nominal case, the active period was modeled as $4.5 \mu \mathrm{g}$ (Earth) $(0.027 \mathrm{ft} / \mathrm{s} / \mathrm{Vhr})$. The quiescent period was modeled with a process noise of $0.45 \mu \mathrm{g}$ (Earth) $(0.0027 \mathrm{ft} / \mathrm{s} / \sqrt{\mathrm{hr}})$. The active periods last for 16 hours per day and the 
quiescent periods last for 8 . The first quiescent period begins 9 hours after TLI and these alternating active/quiescence periods continue for the remainder of the transfer.

There are 12 states associated with the CEV dynamics ( 3 position, 3 velocity, 3 attitude, 3 attitude rate). The maneuvers errors are modeled as biases representing thruster misalignments $(0.01$ degrees $(1 \sigma))$, scale factor $(10$ $\left.\mathrm{ppm}^{\S}(1 \sigma)\right)$, and biases $(0.00328 \mathrm{ft} / \mathrm{s}(1 \sigma))$, along with a random noise of $0.00328 \mathrm{ft} / \mathrm{s}(1 \sigma)$.

It is assumed that the TLI maneuver will have active guidance so that the maneuver errors and cut-off errors will be minimal. As a point of comparison, during Apollo, the S-IVB TLI burn was so accurate that TCM 1 was not performed. It is expected that the residuals associated with this maneuver would be on the order of $1 \mathrm{~m} / \mathrm{s}$.

\section{Sensor Models}

The CEV will have an extensive suite of navigation instruments. While most of them are used for rendezvous, the IMU, GPS receiver, and star tracker are used for all phases of flight, including cislunar operations.

The gyros were modeled after the GG1320 RLGs in the Honeywell MIMU. It has a long-term bias stability of $0.12 \mathrm{deg} / \mathrm{hr}(3 \sigma)$ and a random walk of $0.025 \mathrm{deg} / \mathrm{Vhr}$. There were 18 states associated with the gyros that were modeled. They were: gyro drift Markov (3 states), gyro drift bias (3 states), gyro scale factor Markov (3 states), gyro scale factor bias (3 states), gyro misalignments (6 states). The Markov states had time constants of 1 hour and strength corresponding to the bias states.

The CEV GPS receiver performance was modeled after a Viceroy class GPS receiver. It was assumed to provide deterministic GPS measurements accurate to $50 \mathrm{ft}$ and $0.067 \mathrm{ft} / \mathrm{s}(1 \sigma)$ with updates every 10 seconds. In addition to the measurement nose, the GPS receiver was modeled in the onboard system using 6 Markov states to model the GPS position and velocity errors. The maximum altitude of the GPS receiver was conservatively chosen to be 6475 n.m. $(12,000 \mathrm{~km})$.

The star tracker was modeled after a Goodrich HD1003 and provides attitude updates in the form of an inertialto-sensor quaternion every 10 minutes. The measurement accuracy was 62 arc-seconds $(3 \sigma)$. The misalignment was 36 arc-seconds $(3 \sigma)$.

There are infrequent updates to the navigation states of the CEV. These are performed prior to each maneuver. The accuracy of the ground updates is listed in Table 3, reflective of ground tracking capabilities during the Apollo 10 mission $^{* *}$. It is expected that today's capabilities are comparable due to the fact that there are few tracking stations available and the geometry of those is not as favorable; however technology has advanced in the intervening years. Table 1 contains the ground-tracking accuracies used in this analysis. In upcoming years, further analysis will be performed to better characterize the expected cislunar navigation accuracies.

\begin{tabular}{|c|c|c|c|c|c|c|c|}
\hline $\begin{array}{c}\text { 1 } \boldsymbol{\sigma} \text { Error } \\
\text { Component }\end{array}$ & TCM & \multicolumn{1}{c}{ TCM } & \multicolumn{1}{c}{ TCM } & \multicolumn{1}{c|}{ TCM } & LOI & LOI & LOI \\
Radial $(\mathrm{U})$ & $482 \mathrm{ft}$ & $\mathbf{2}$ & $\mathbf{3}$ & $\mathbf{4}$ & $\mathbf{1}$ & $\mathbf{2}$ & $\mathbf{3}$ \\
\hline Downrange $(\mathrm{V})$ & $7263 \mathrm{ft}$ & $13549 \mathrm{ft}$ & $1934 \mathrm{ft}$ & $4031 \mathrm{ft}$ & $3032 \mathrm{ft}$ & $516 \mathrm{ft}$ & $3032 \mathrm{ft}$ \\
\hline Crossrange $(W)$ & $13919 \mathrm{ft}$ & $10020 \mathrm{ft}$ & $14228 \mathrm{ft}$ & $1851 \mathrm{ft}$ & $5051 \mathrm{ft}$ & $18002 \mathrm{ft}$ & $5051 \mathrm{ft}$ \\
\hline Radial Rate $(\mathrm{U}-\mathrm{dot})$ & $0.058 \mathrm{ft} / \mathrm{s}$ & $0.82 \mathrm{ft} / \mathrm{s}$ & $0.015 \mathrm{ft} / \mathrm{s}$ & $0.027 \mathrm{ft} / \mathrm{s}$ & $3.241 \mathrm{ft} / \mathrm{s}$ & $45571 \mathrm{ft}$ & $8109 \mathrm{ft}$ \\
\hline Downrange Rate $(\mathrm{V}-\mathrm{dot})$ & $0.214 \mathrm{ft} / \mathrm{s}$ & $0.39 \mathrm{ft} / \mathrm{s}$ & $0.064 \mathrm{ft} / \mathrm{s}$ & $0.008 \mathrm{ft} / \mathrm{s}$ & $1.177 \mathrm{ft} / \mathrm{s}$ & $0.343 \mathrm{ft} / \mathrm{s}$ & $1.177 \mathrm{ft} / \mathrm{s}$ \\
\hline Crossrange Rate $(W-d o t)$ & $0.318 \mathrm{ft} / \mathrm{s}$ & $0.49 \mathrm{ft} / \mathrm{s}$ & $0.061 \mathrm{ft} / \mathrm{s}$ & $0.042 \mathrm{ft} / \mathrm{s}$ & $7.300 \mathrm{ft} / \mathrm{s}$ & $1.010 \mathrm{ft} / \mathrm{s}$ & $7.300 \mathrm{ft} / \mathrm{s}$ \\
\hline
\end{tabular}

Table 1: Translunar Ground Update Navigation Accuracy Parameters

\section{B. Design Reference Mission Trajectories}

The translunar trajectory was designed to deliver the CEV to a low lunar orbit five days after the TLI (TransLunar Injection). There is a three-burn Lunar Orbit Insertion (LOI) maneuver sequence to insert the CEV/LSAM stack into a near-polar Low Lunar Orbit (LLO). For the return trip, a three-burn TEI sequence essentially unwinds the LOI sequence and inserts the CEV into a departure hyperbola that results in a desired Entry Interface Point. The maneuver times, their objectives and the nominal $\Delta V \mathrm{~s}$ are given in Table 2. The corresponding transEarth (Moonto-Earth) maneuver information is listed in Table 3, with the times referenced to the $\mathrm{TEI}_{1}$ maneuver

\footnotetext{
$\S$ Parts per million

*** Lewis, L.R., et.al., Apollo 10 Spacecraft Dispersion Analysis, Volume 1- Navigation Error Analysis, MSC Internal Note No. 69-FM-83, April 14, 1969.
} 


\begin{tabular}{|c|c|c|c|}
\hline $\begin{array}{l}\text { Maneuver } \\
\text { Type }\end{array}$ & $\begin{array}{c}\text { Maneuver Time } \\
\text { (hrs) }\end{array}$ & Maneuver Objective & Nominal $\Delta V(\mathbf{f t} / \mathbf{s})$ \\
\hline$T L I$ & 0 & $\begin{array}{l}\text { Target for } h_{p} \text {, Energy, Inclination at } \\
\text { Pericynthion }\end{array}$ & 10,308 \\
\hline$\overline{T C M_{1}}$ & 6 hours (TLI +6 hours) & $\begin{array}{c}\text { Target for } h_{p} \text {, Energy, Inclination at } \\
\text { Pericynthion }\end{array}$ & 0.0 \\
\hline $\mathrm{TCM}_{2}$ & 24 hours (TLI +1 day) & $\begin{array}{c}\text { Target for } h_{p} \text {, Energy, Inclination at } \\
\text { Pericynthion }\end{array}$ & 0.0 \\
\hline $\mathrm{TCM}_{3}$ & 72 hours (LOI -1 day) & $\begin{array}{c}\text { Target for } h_{p} \text {, Energy, Inclination at } \\
\text { Pericynthion }\end{array}$ & 0.0 \\
\hline $\mathrm{TCM}_{4}$ & $\begin{array}{c}90 \text { hours (LOI }-6 \\
\text { hours) }\end{array}$ & $\begin{array}{c}\text { Target for } h_{p} \text {, Energy, Inclination at } \\
\text { Pericynthion }\end{array}$ & 0.0 \\
\hline$L O I_{1}$ & 96 hours & Apocynthion Altitude & 826 \\
\hline $\mathrm{LOI}_{2}$ & 99.5 hours & $\begin{array}{c}\text { Wedge Angle, Energy, Pericynthion } \\
\text { altitude }\end{array}$ & 216 \\
\hline $\mathrm{LOI}_{3}$ & 120 hours & $\begin{array}{c}\text { Apocynthion Altitude } \\
\text { (circularization) }\end{array}$ & 1856 \\
\hline
\end{tabular}

Table 2: Translunar Maneuver Information

\begin{tabular}{|c|c|c|c|}
\hline $\begin{array}{c}\text { Maneuver } \\
\text { Type }\end{array}$ & $\begin{array}{c}\text { Maneuver Time (hrs) } \\
\text { (Past TEI } \text { TE }_{1}\end{array}$ & Maneuver Objective & Nominal $\Delta V(\mathrm{ft} / \mathrm{s})$ \\
\hline$T E I_{I}$ & 2.68 & Raise Apocynthion altitude & 6147 \\
\hline$T E I_{2}$ & 15.16 & $\begin{array}{c}\text { Wedge Angle Change, } \\
\text { Energy Adjust, Pericynthion } \\
\text { Altitude Adjust }\end{array}$ & 1529 \\
\hline $\mathrm{TEI}_{3}$ & 24.05 & $\begin{array}{c}\text { Target for Entry FPA, } \\
\text { Azimuth, Latitude, Longitude }\end{array}$ & 3623 \\
\hline$T C M_{5}$ & 42.05 hours (TEI +18 hours) & $\begin{array}{c}\text { Target for Entry FPA, } \\
\text { Azimuth, Latitude }\end{array}$ & 0.0 \\
\hline$T C M_{6}$ & 92.03 hours (EI - 16 hours) & $\begin{array}{l}\text { Target for Entry FPA, } \\
\text { Azimuth, Latitude }\end{array}$ & 0.0 \\
\hline$T C M_{7}$ & 103.03 hours (EI -5 hours) & $\begin{array}{l}\text { Target for Entry FPA, } \\
\text { Azimuth, Latitude }\end{array}$ & 0.0 \\
\hline
\end{tabular}

Table 3: TransEarth Maneuver Information

\section{Translunar Navigation and Dispersion Analysis}

In order to analyze the overall system performance, several trades were performed to ascertain the sensitivity to factors such as frequent ground updates, maneuver execution errors and vehicle noise. Whereas analysis has been 
performed for various ground update frequencies, the best performance is obtained, as expected, for the case of frequent ground updates. Figure 1 shows the position and velocity navigation errors for the out-bound trajectory. Noted on the plots is the effect of ground navigation updates on the on-board navigation errors.
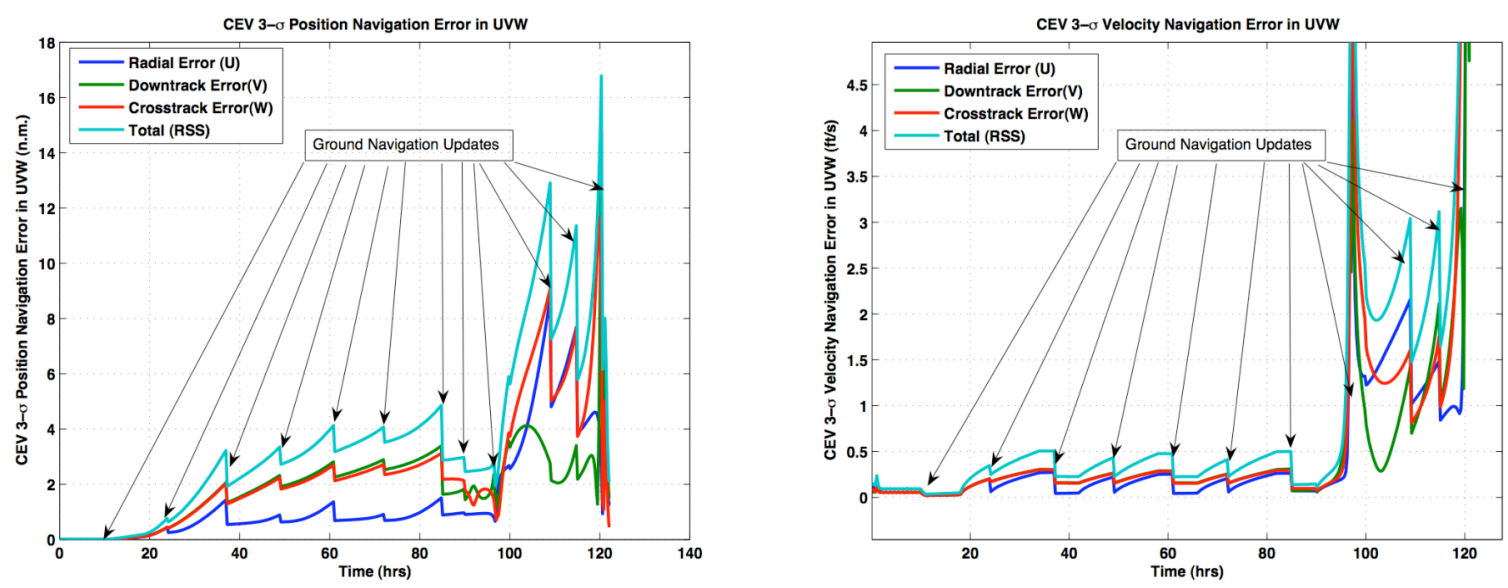

Figure 1: On-board Position and Velocity Navigation Errors

\section{Navigation Error Analysis}

The UVW coordinate system, while useful in certain applications (such as rendezvous) are less useful for orbits with high eccentricity (such as the Earth-Moon and Moon-Earth transfers). As such it is useful to find other metrics for analyzing navigation (and dispersion) errors. One of the most useful metrics is to propagate the (current) navigation errors to the final epoch (usually periapsis or entry-interface). For covariance analysis, this is done by means of the state transition matrix and a transformation from inertial coordinates to the parameters of interest. For the translunar trajectory, the quantities of interest are the pericynthion (closest approach) altitude, inclination, and energy. Figure 2 contains a plot of the navigation error mapped to pericynthion altitude, with the right plot containing the zoomed in mapped pericynthion altitude error. Figure 3 contains the mapped pericynthion inclination error and the $C_{3}$ error $\left(C_{3}=-\mu / 2 a\right)$.
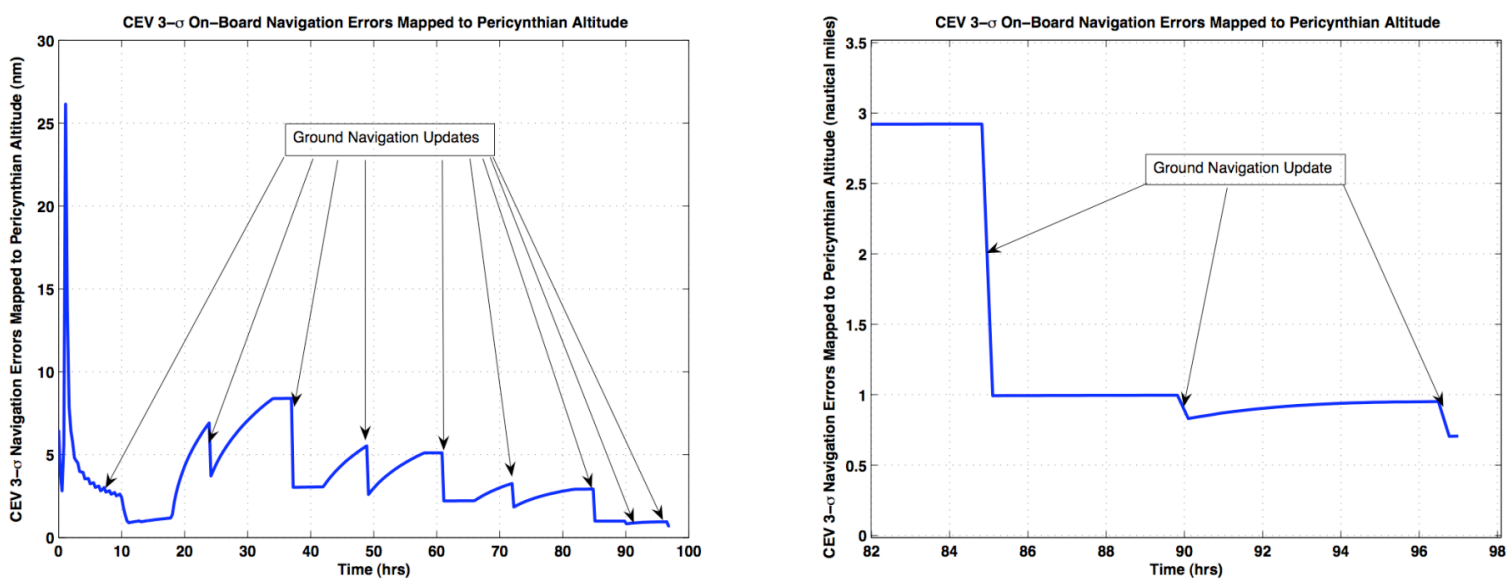

Figure 2: Translunar coast navigation errors mapped to pericynthion altitude 

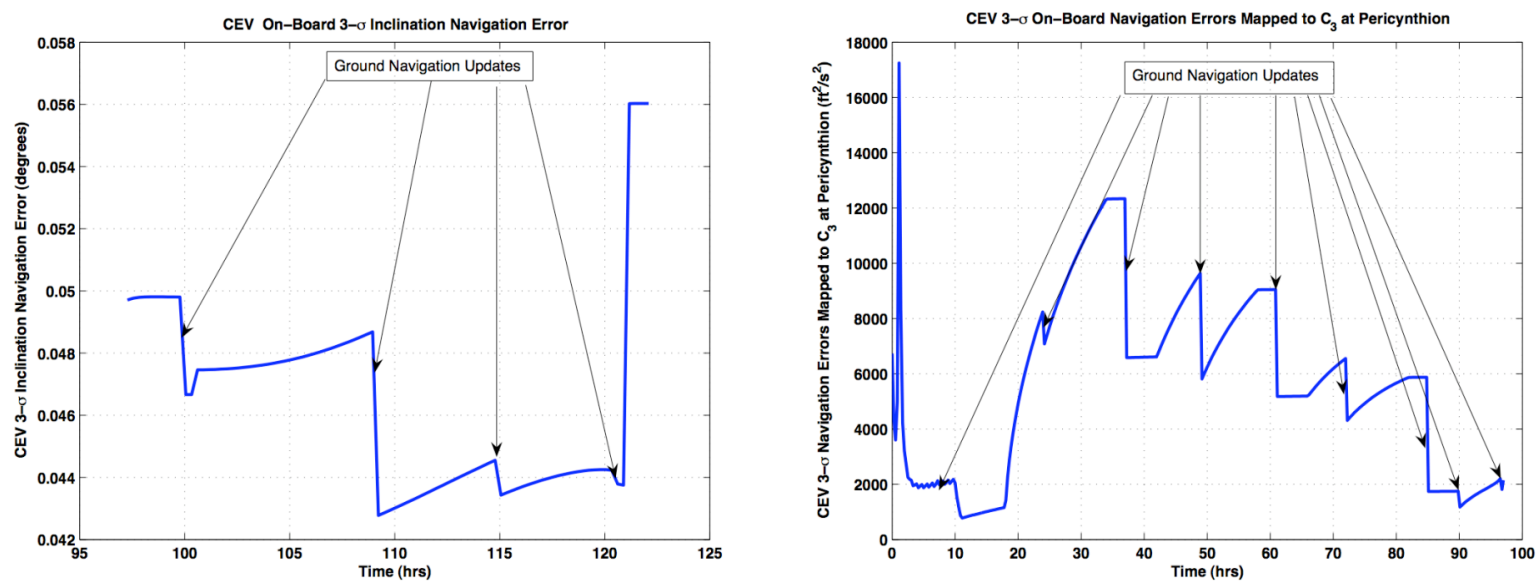

Figure 3: Translunar coast navigation errors mapped to Pericynthion Inclination and $\boldsymbol{C}_{3}$

Figure 4 shows the periapsis altitude and semi-major axis navigation error after the first LOI maneuver, when the $\mathrm{CEV}$ is captured into lunar orbit. Figure 5 shows the inclination and longitude of the ascending node navigation error upon capture in lunar orbit.
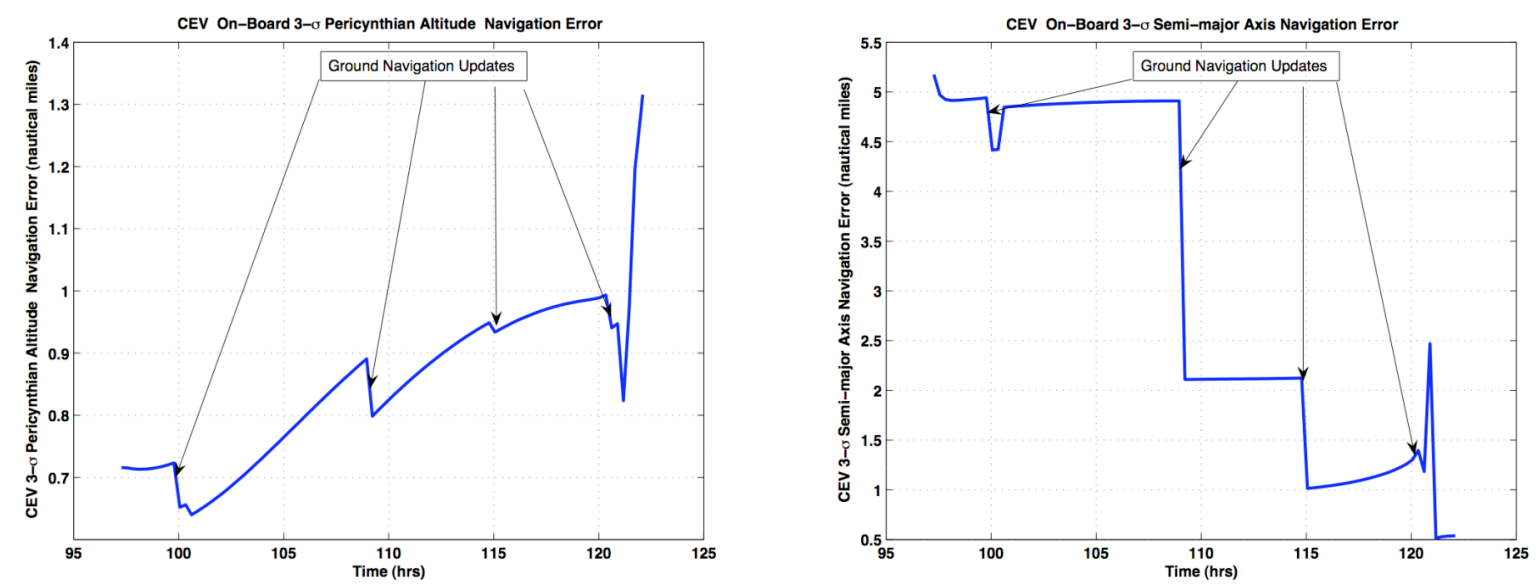

Figure 4: Periapsis alitude and Semi-major axis navigation errors
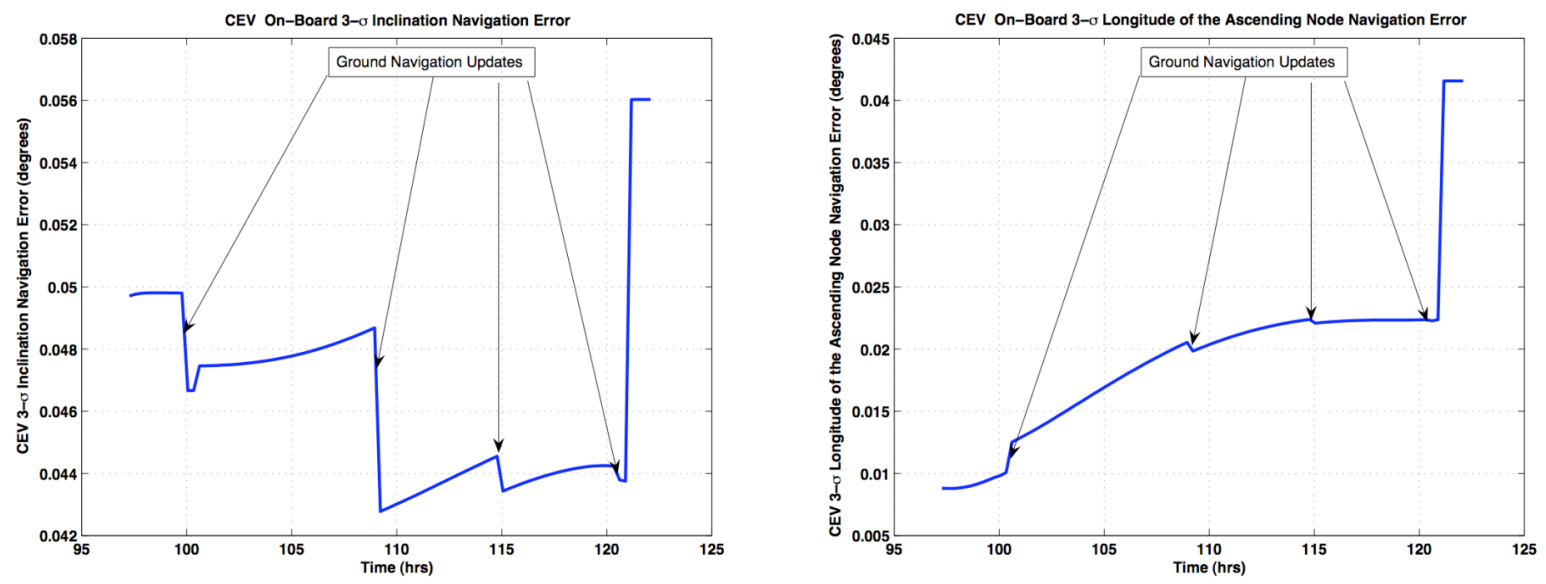

Figure 5: Inclination and Longitude of the Ascending Node navigation errors 


\section{Dispersion Analysis}

Linear covariance analysis also provides the analyst with trajectory dispersions (defined as the difference between the actual trajectory and the nominal trajectory). As with the navigation errors, the translunar trajectory dispersion errors were mapped to pericynthion and expressed in terms of the quantities of interest at pericynthion.
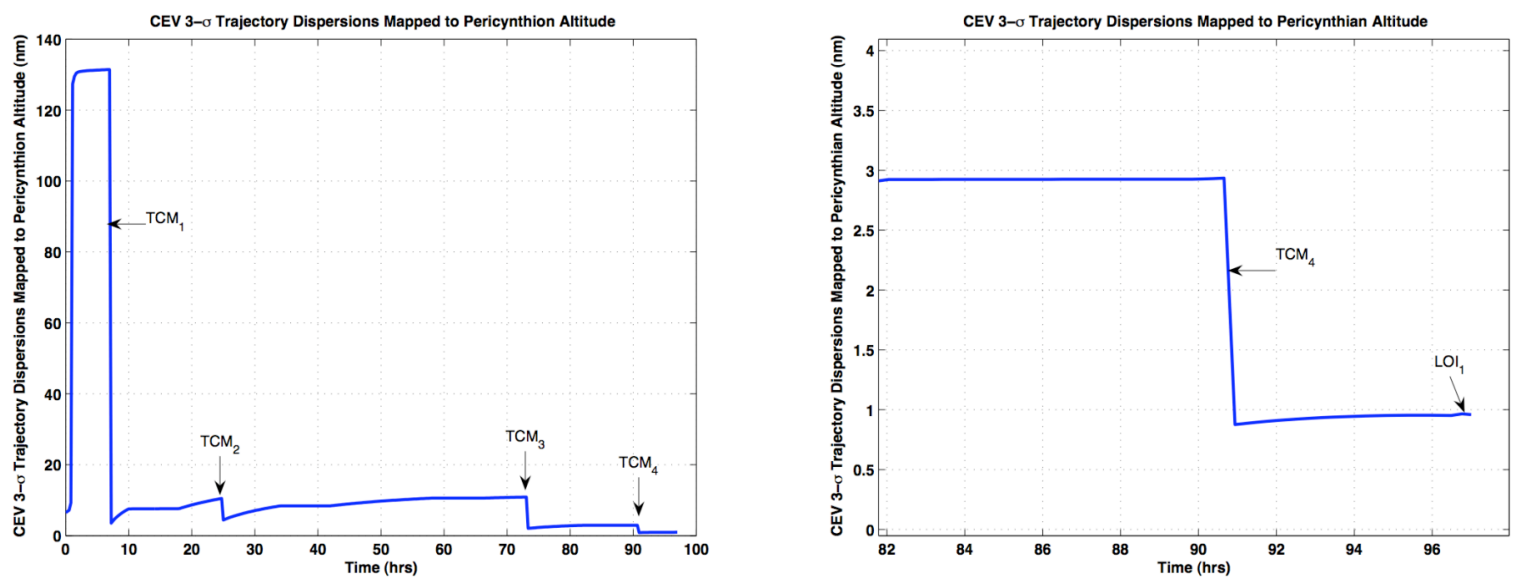

Figure 6: Translunar Trajectory Dispersions Mapped to Pericynthion Altitude Errors

Figures 8 and 9 contain the trajectory dispersions mapped to orbital quantities of the post-insertion orbit. One can see that the dispersions after the sequence of insertion maneuvers are completed are quite good and well-contained. This bodes well for the translunar and insertion phase of the mission, assuming that the ground navigation system is kept to the accuracy of the Apollo tracking station network. Finally, Table 4 contains the $\Delta V$ dispersion statistics associated with the maneuvers along this trajectory and Table 5 contains the final post-insertion orbit.
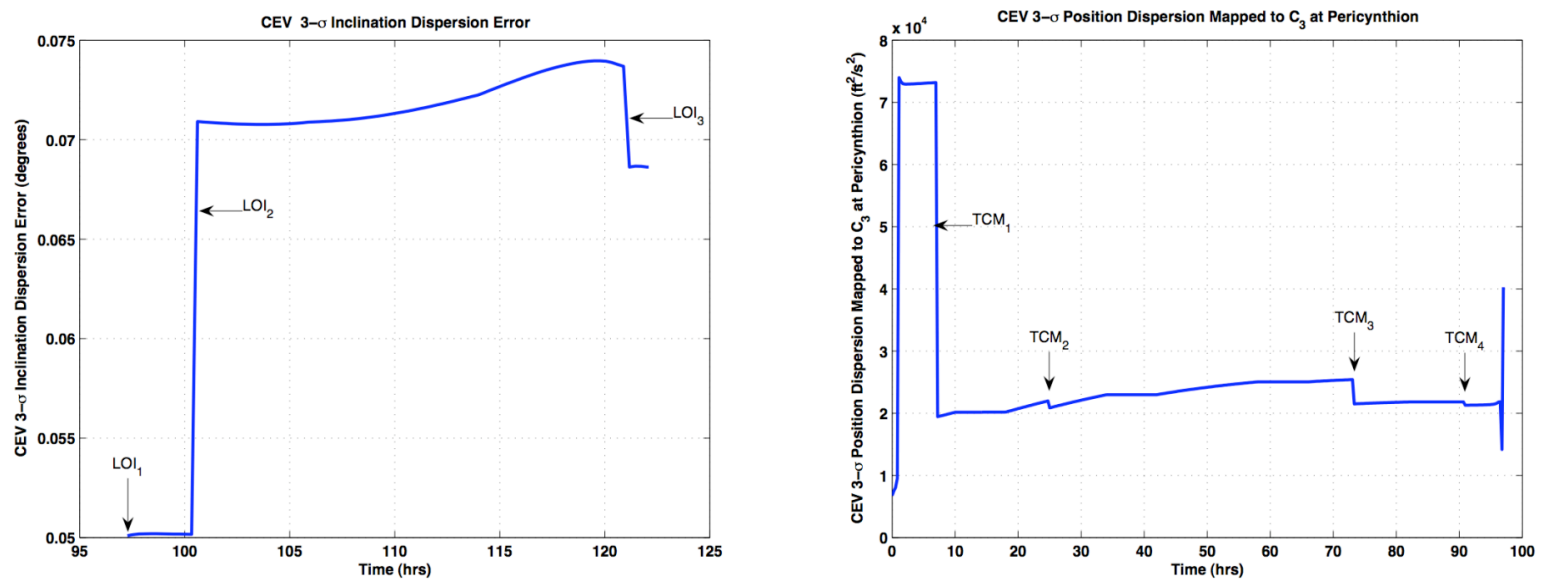

Figure 7: Translunar Trajectory Dispersions Mapped to Pericynthion Inclination and C3 Errors 

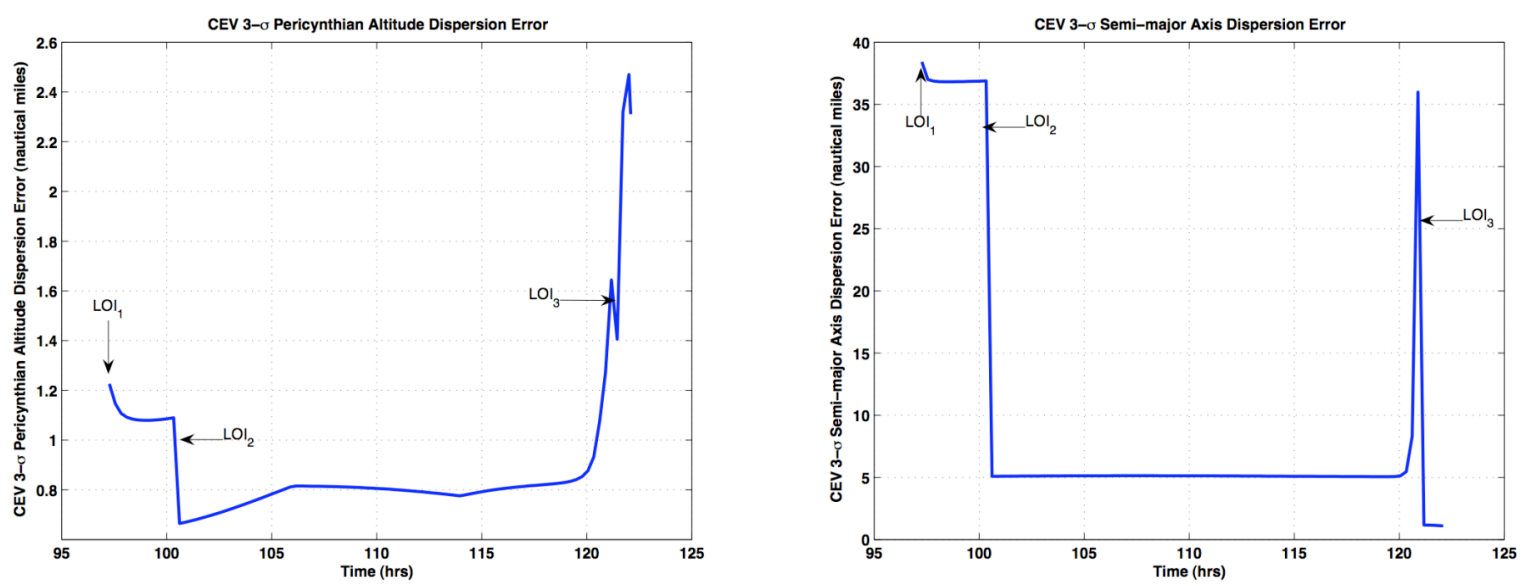

Figure 8: Post-insertion Trajectory Dispersions Mapped to Semi-major axis and Pericynthion Altitude
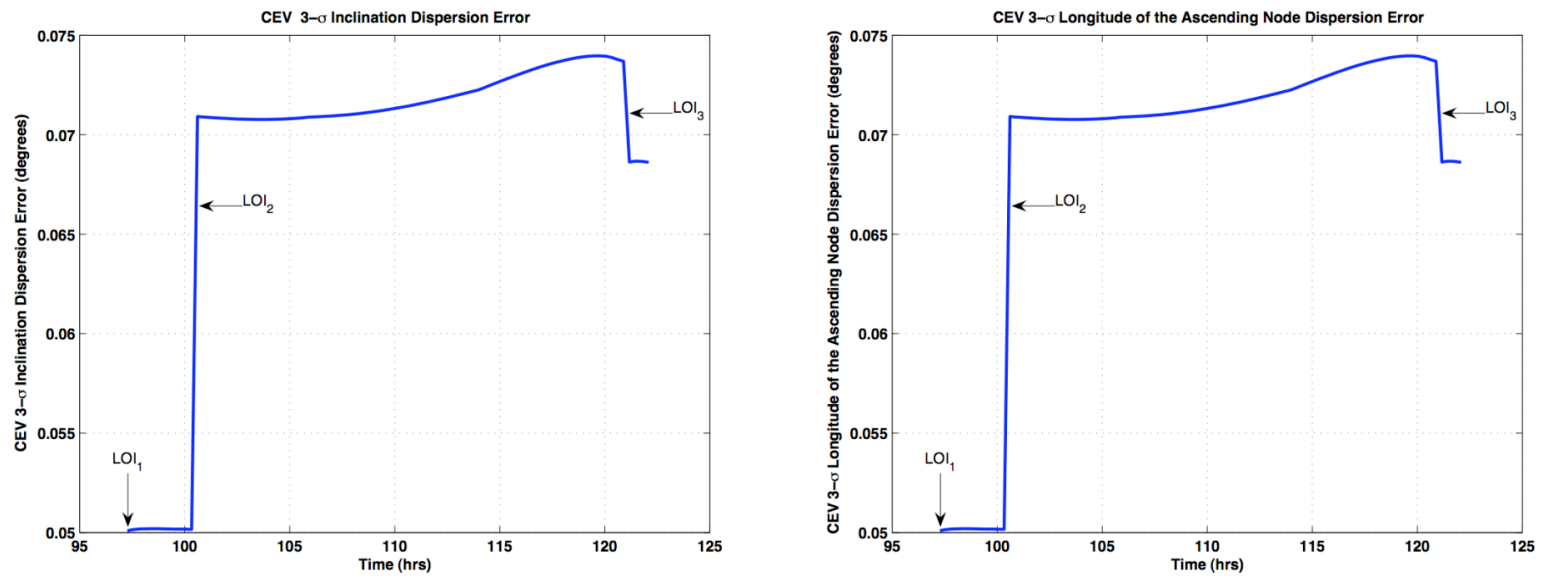

Figure 9: Post-Insertion Trajectory Dispersions Mapped to Inclination and Longitude of the Ascending Node

\begin{tabular}{|c|c|c|}
\hline Maneuver Type & $\begin{array}{c}\text { Nominal } \boldsymbol{\Delta} \boldsymbol{V} \\
(\mathbf{f t} / \mathbf{s})\end{array}$ & $\begin{array}{c}\boldsymbol{\sigma}_{\boldsymbol{\Delta}} \\
(\mathbf{f t} / \mathbf{s})\end{array}$ \\
\hline $\mathrm{TCM}_{1}$ & 0 & 0.997 \\
\hline $\mathrm{TCM}_{2}$ & 0 & 0.140 \\
\hline $\mathrm{TCM}_{3}$ & 0 & 0.496 \\
\hline $\mathrm{TCM}_{4}$ & 0 & 0.423 \\
\hline $\mathrm{LOI}_{1}$ & 825.997 & 9.360 \\
\hline $\mathrm{LOI}_{2}$ & 215.725 & 3.485 \\
\hline $\mathrm{LOI}_{3}$ & 1855.804 & 61.297 \\
\hline
\end{tabular}

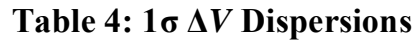

\begin{tabular}{|c|c|}
\hline $\begin{array}{c}\text { Error } \\
\text { Component }\end{array}$ & $\mathbf{( 1 \sigma )}$ \\
\hline Semi-major Axis & $677.2 \mathrm{ft}$ \\
\hline Periapsis Altitude & $1426.5 \mathrm{ft}$ \\
\hline Inclination & $0.0229 \mathrm{deg}$ \\
\hline Longitude of the Ascending Node & $0.0206 \mathrm{deg}$ \\
\hline
\end{tabular}

Table 5: $1 \sigma$ Dispersions at the Conclusion of the LOI phase $\left(t_{\mathrm{LOI3}}+1\right.$ hour) 


\section{Trans-Earth Navigation and Dispersion Analysis}

As in the case of the translunar analysis, both the navigation and the trajectory dispersions will be analyzed. The navigation errors for the entire phase (in terms of UVW) are illustrated in Figure 10.
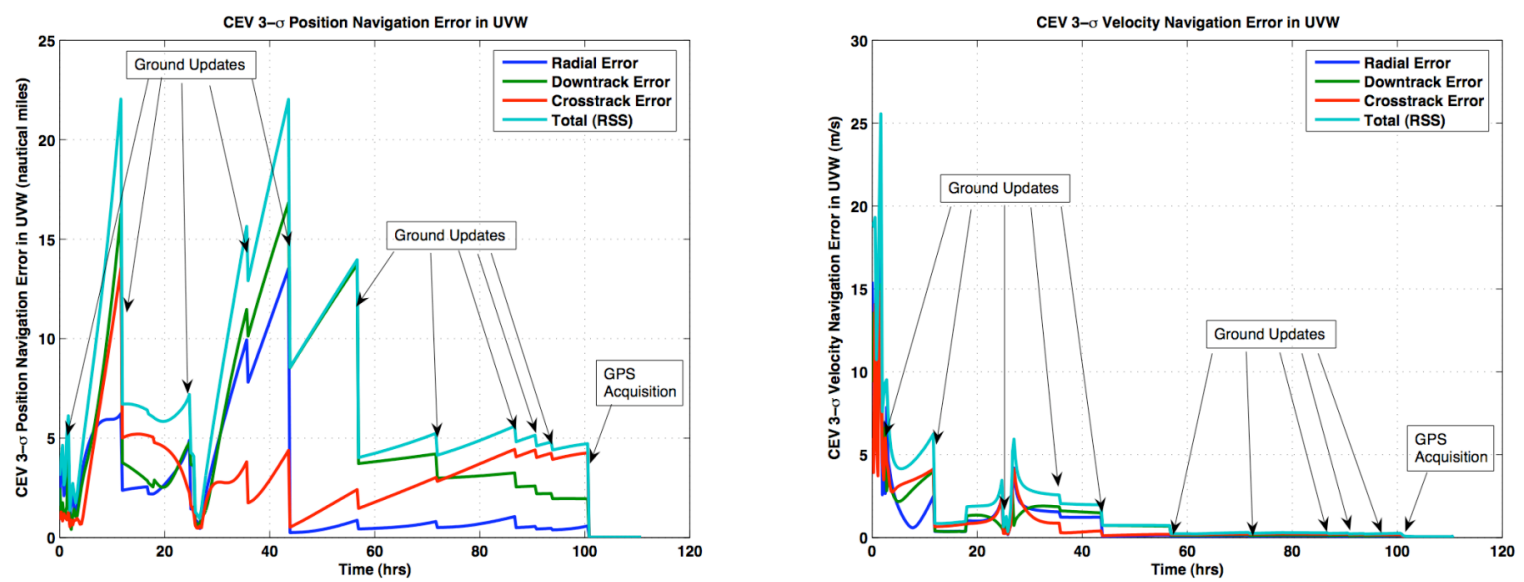

Figure 10: TransEarth On-board Navigation Errors

\section{Navigation Error Analysis}

As in the previous section, the navigation errors will be analyzed as a function of the sub-phase: lunar orbit and trans-Earth. This time, however, we look at the TransEarth Injection phase (which includes the TransEarth Injection (TEI) maneuvers) followed by the TransEarth coast phase. Figure 11 contains the plots of the pericynthion altitude and semi-major axis navigation errors; Figure 12 contains the inclination and longitude of the ascending node navigation errors. One can clearly see the effect of the ground (Earth-based) navigation updates on the navigation state. Figures 13 and 14 contain the on-board navigation errors mapped to the entry-interface point $(400,000 \mathrm{ft}$ above the surface of the earth). One can see the effect of the ground-based navigation updates and the effect of GPS acquisition. For this analysis, it was assumed that GPS was acquired $1 / 2$ way through the GPS shell $(\sim 12,000 \mathrm{~km}$ above the surface).
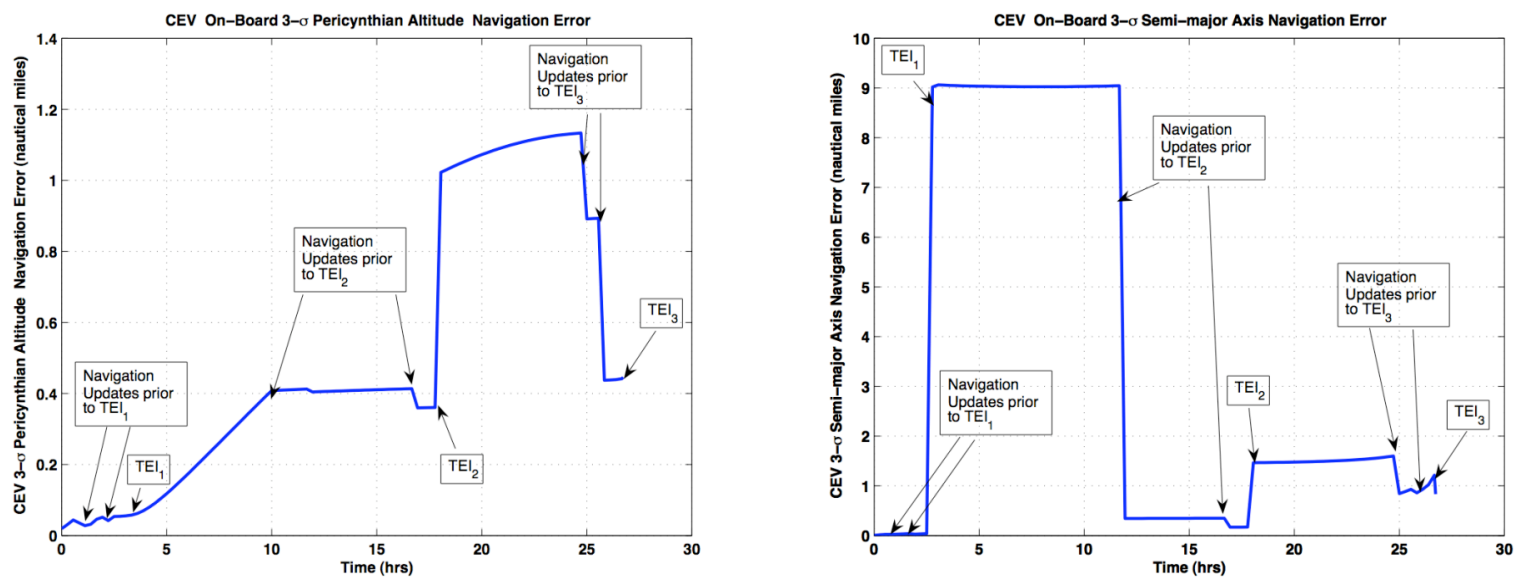

Figure 11: Pericynthion Altitude and Semi-major axis navigation errors for the Trans-Earth Injection Phase 

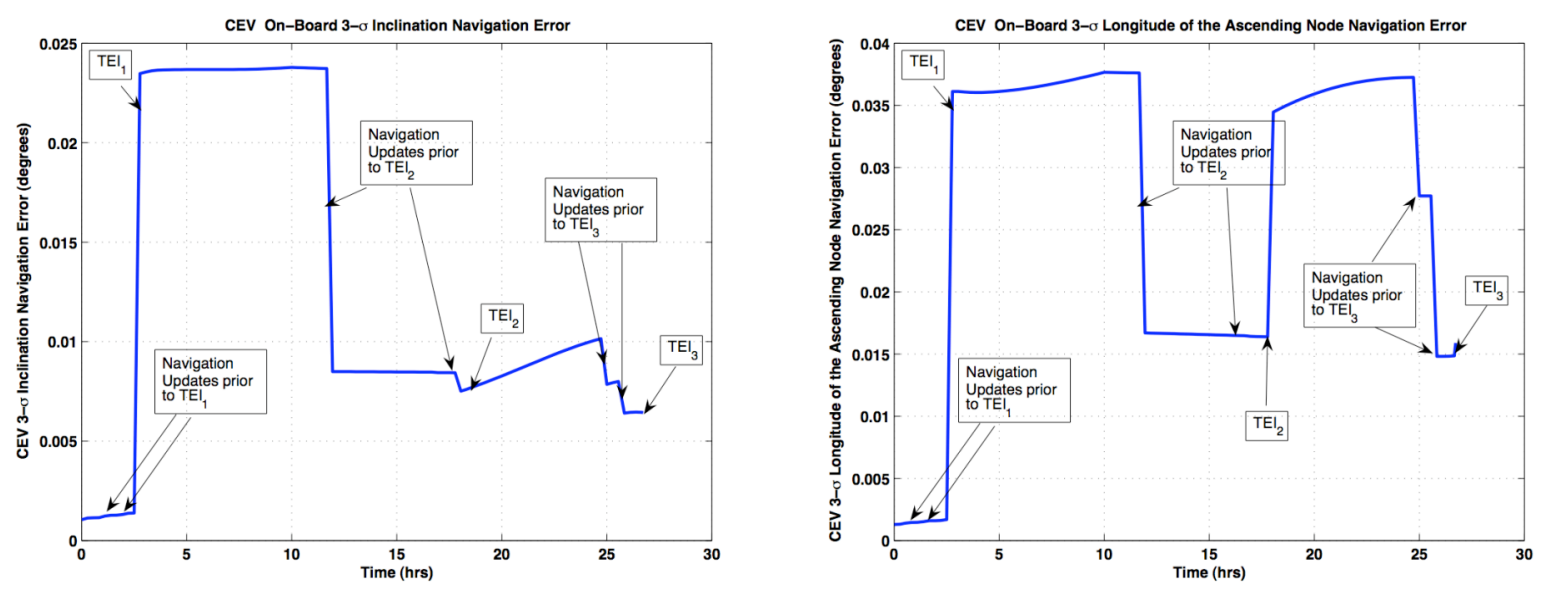

Figure 12 : Inclination and longitude of the ascending node navigation errors for the TEI Phase
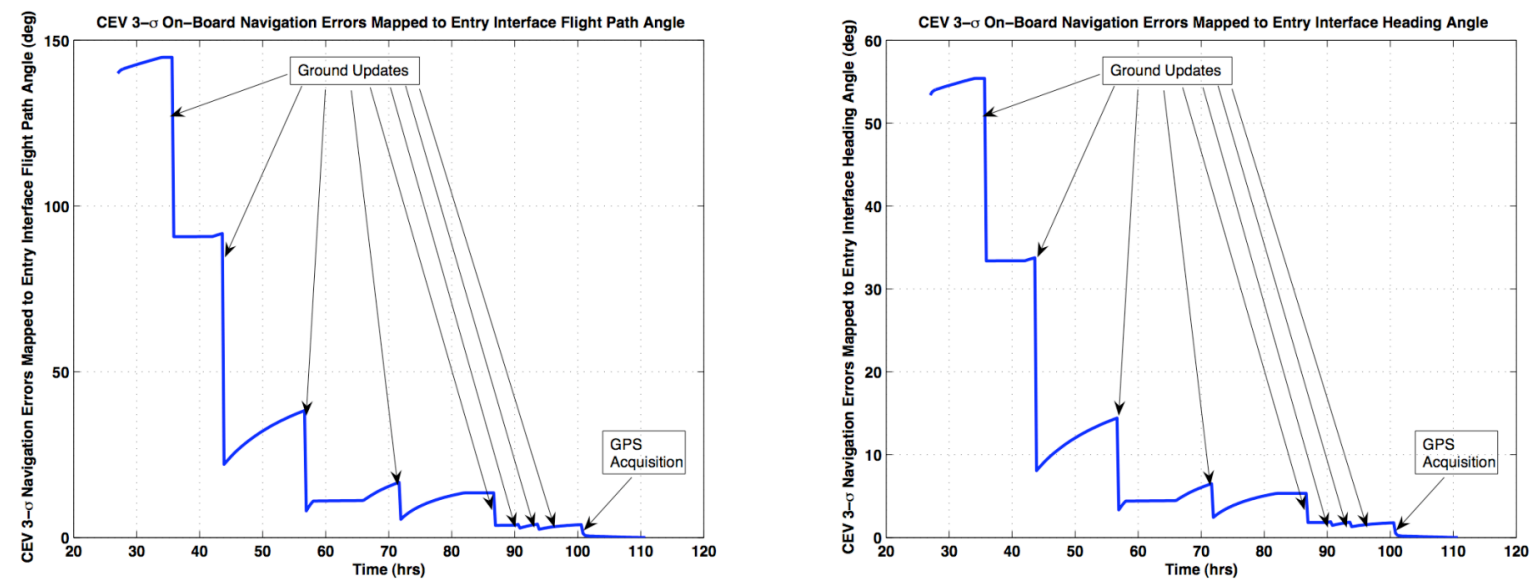

Figure 13: TranslEarth coast navigation errors mapped to entry interface flight path angle and heading angle
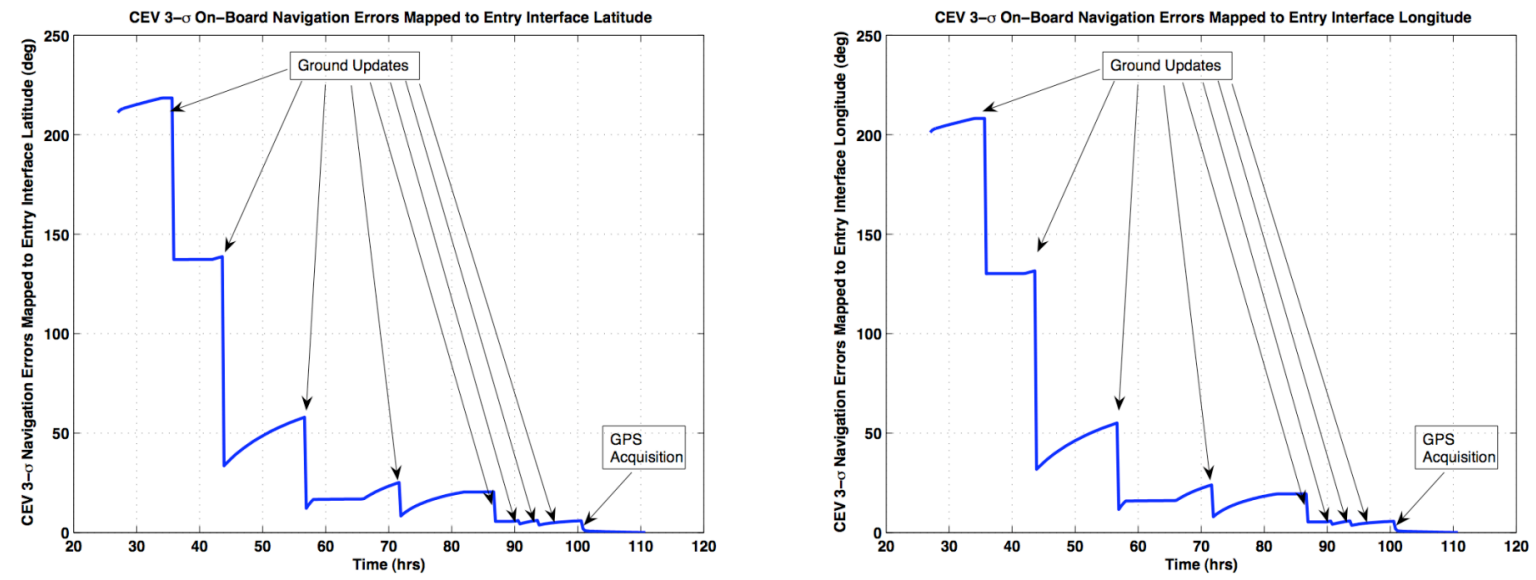

Figure 14: TransEarth coast navigation errors mapped to entry interface latitude and longitude

\section{2. $\quad$ TransEarth Dispersion Analysis}

The trajectory dispersions for the TEI maneuver phase (the phase between $\mathrm{TEI}_{1}$ and $\mathrm{TEI}_{3}$ ) of the pericynthion altitude and semi-major axis errors are shown in Figure 15 and the inclination and longitude of the ascending node 
dispersions are illustrated in Figure 16. For the transEarth coast phase, Figure 17 shows the trajectory dispersions mapped to the entry interface flight-path angle and heading angle and Figure 18 shows the trajectory dispersions mapped to entry latitude and longitude.

Finally, Table 6 has the entry-interface trajectory dispersions expressed in terms of entry flight path angle, heading angle, latitude and longitude and Table 7 has the 1s DV statistics. One can see that the dispersions at TEI3 are the largest, but that is anticipated due to the large size of that maneuver. It is primarily caused by navigation errors during the prior maneuvers. The TCM dispersions for the transEarth coast phase are well contained.
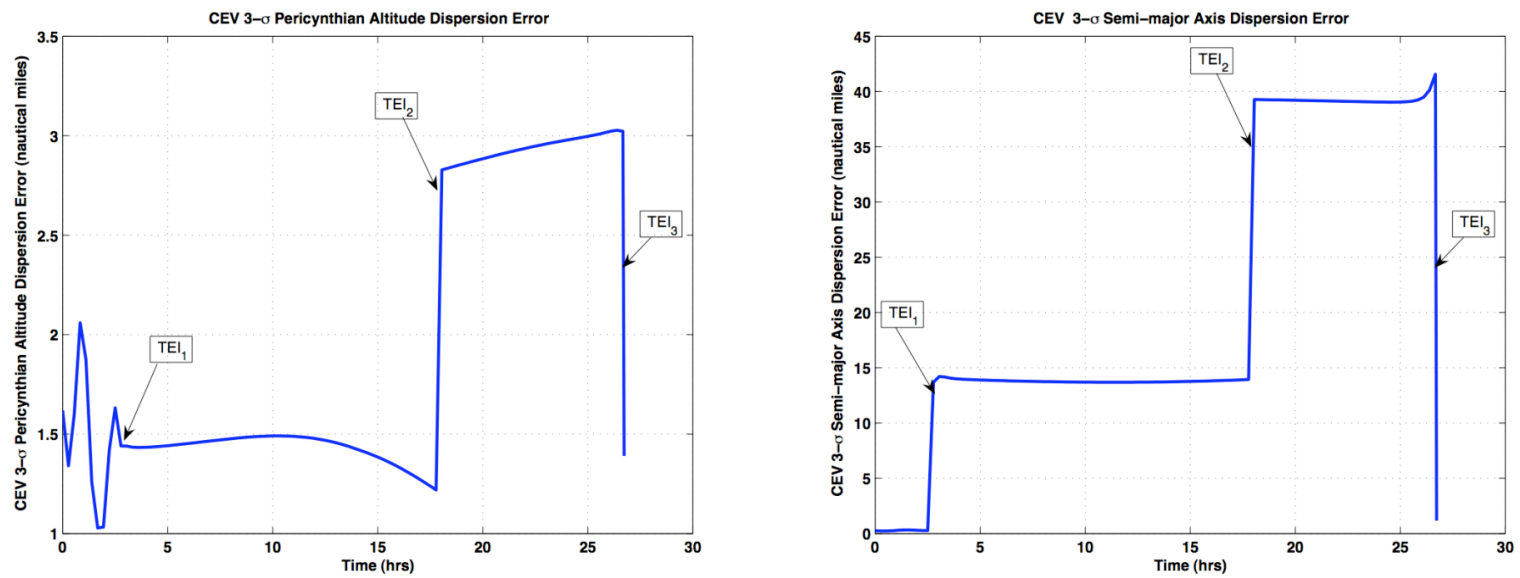

Figure 15: Pericynthion altitude and semi-major axis dispersion errors for the TEI maneuver phase
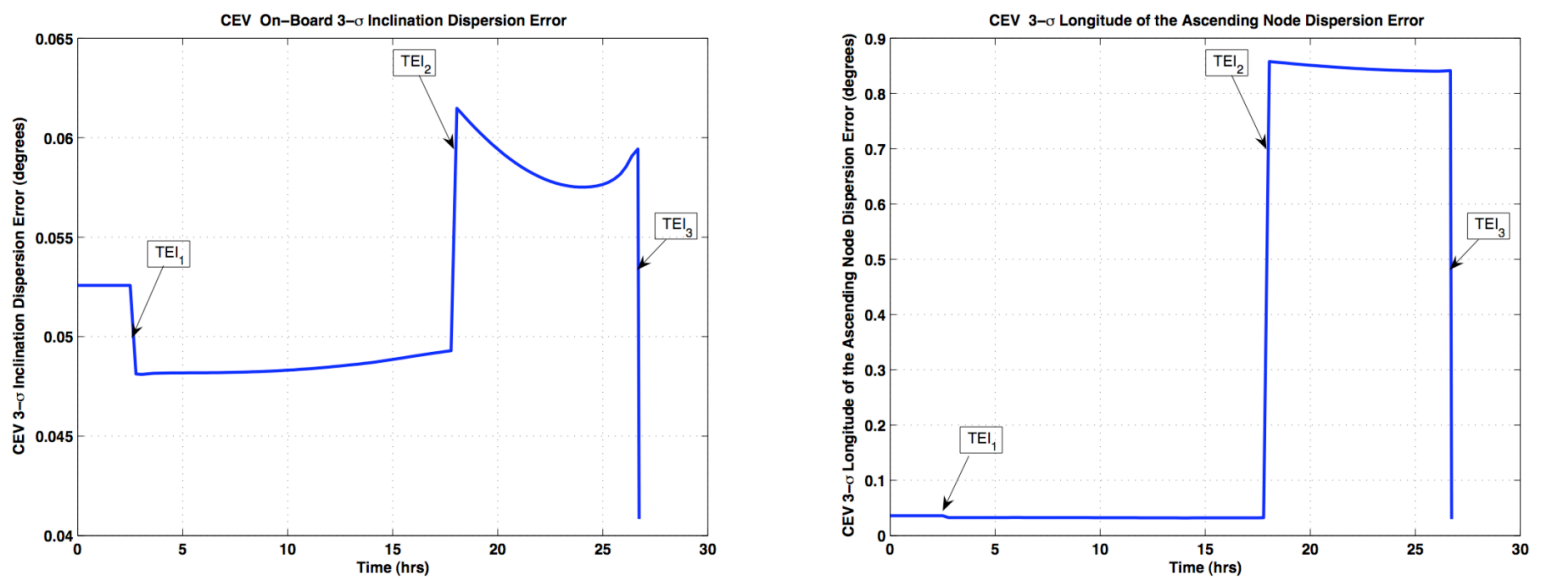

Figure 16: Inclination and longitude of the ascending node dispersion errors for the TEI phase 

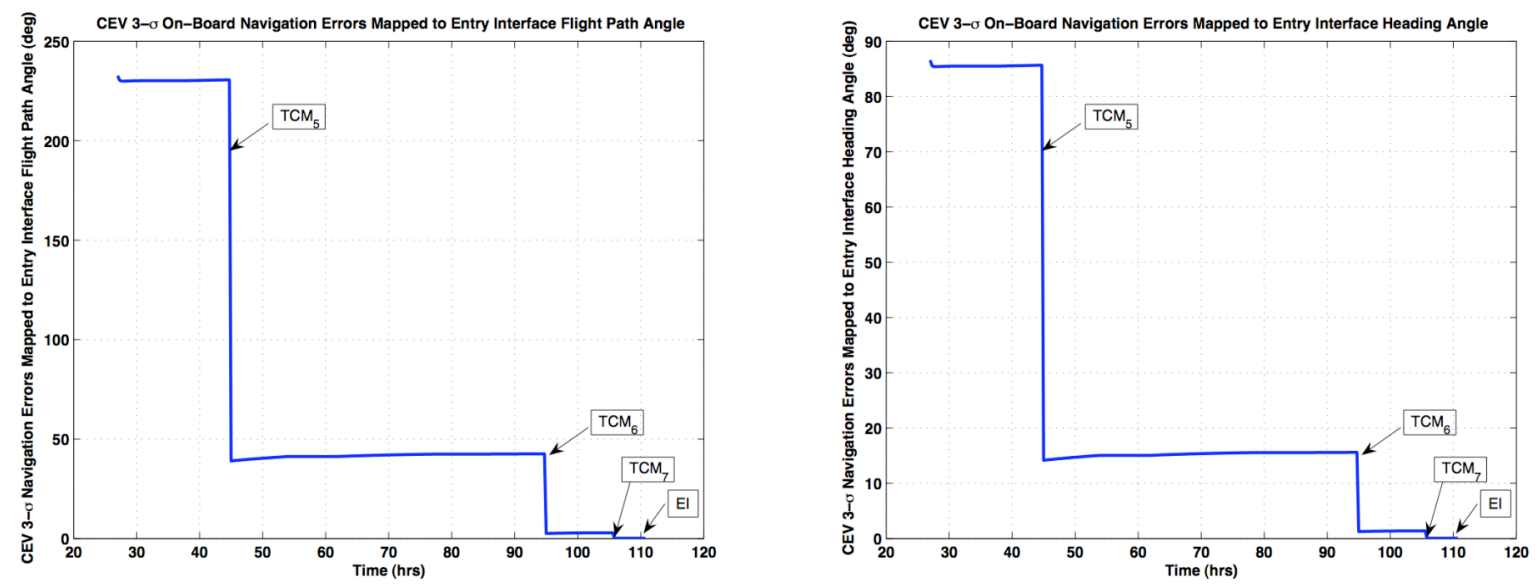

Figure 17: TransEarth coast trajectory dispersion errors mapped to entry interface flight path angle and heading angle
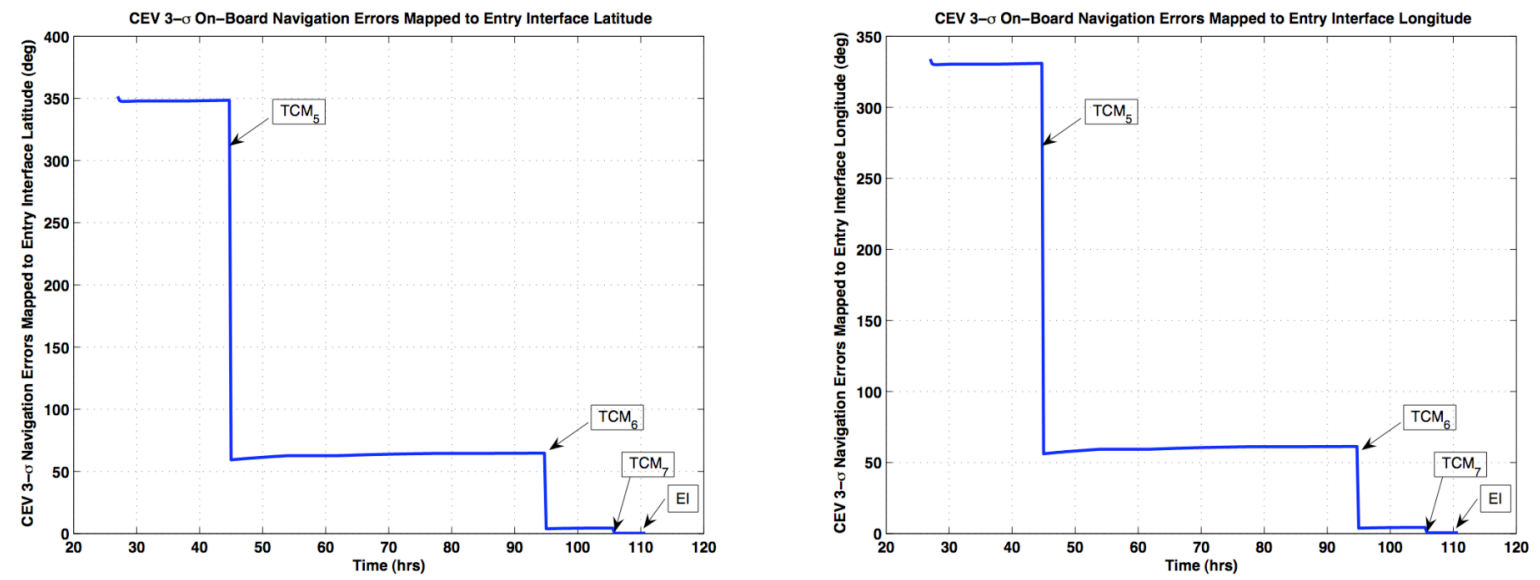

Figure 18: TransEarth coast trajectory dispersion errors mapped to entry interface latitude and longitude

\begin{tabular}{|c|c|}
\hline $\begin{array}{c}\text { Error } \\
\text { Component }\end{array}$ & $\begin{array}{c}\text { Entry Dispersion } \\
(\mathbf{1 \sigma})\end{array}$ \\
\hline Flight Path Angle & $0.0486 \mathrm{deg}$ \\
\hline Heading Angle & $0.0234 \mathrm{deg}$ \\
\hline Latitude & $0.0758 \mathrm{deg}$ \\
\hline Longitude & $0.2089 \mathrm{deg}$ \\
\hline
\end{tabular}

Table 6: 1 $\sigma$ Dispersions at Entry Interface

\begin{tabular}{|c|c|c|}
\hline Maneuver Type & $\begin{array}{c}\text { Nominal } \boldsymbol{\Delta} \boldsymbol{V} \\
(\mathbf{f t} / \mathbf{s})\end{array}$ & $\begin{array}{c}\boldsymbol{\sigma}_{\boldsymbol{\Delta V}} \\
(\mathbf{f t} / \mathbf{s})\end{array}$ \\
\hline $\mathrm{TEI}_{1}$ & 1873.634 & 8.376 \\
\hline $\mathrm{TEI}_{2}$ & 465.996 & 8.647 \\
\hline $\mathrm{TEI}_{3}$ & 1104.216 & 25.165 \\
\hline $\mathrm{TCM}_{5}$ & 0 & 0.713 \\
\hline $\mathrm{TCM}_{6}$ & 0 & 0.869 \\
\hline $\mathrm{TCM}_{7}$ & 0 & 0.442 \\
\hline
\end{tabular}

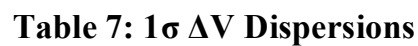




\section{Conclusion}

This paper has documented the analysis and design performed for the Orion translunar and transEarth phases. The analysis has demonstrated that if the Apollo-era tracking and data network navigation data is available, excellent $\Delta \mathrm{V}$ dispersions and navigation performance is obtained. Future analysis will further flesh out the details as the design matures.

\section{References}

${ }^{1} \mathrm{Hu}, \mathrm{H}$. and Chambers, R., "Orion GN\&C Overview and Architecture," AIAA Guidance, Navigation, and Control Conference, AIAA-07-6678, Hilton Head, S.C., August 2007.

${ }^{2}$ Condon, J. and Buches, B., "Orion Mission Design and Analysis," AIAA Guidance, Navigation, and Control Conference, AIAA-07-6680, Hilton Head, S.C., August 2007. 